\title{
Unilateral hilar or paratracheal adenopathy in sarcoidosis: a study of 38 cases
}

\author{
RICHARDW. SPANN, EDWARD C. ROSENOW, III, \\ RICHARD A. DEREMEE, and W. EUGENE MILLER
}

Mayo Clinic, Rochester, Minnesota

\begin{abstract}
The diagnosis of pulmonary sarcoidosis should be considered when unilateral hilar enlargement or a paratracheal mass is present. With this diagnosis in mind, a scalene node biopsy or mediastinoscopy may prevent unnecessary thoracotomy. It is believed that the unilateral stage is only an evanescent stage before the development or regression of bilateral hilar lymphadenopathy.
\end{abstract}

Sarcoidosis is a disease of unknown cause that is characterized by the presence of non-caseous granuloma. The diagnosis is made whenever this histological finding occurs in the absence of other known causes of non-caseating granulomas and whenever compatible clinical and radiographic findings are present. The usual chest radiographic findings in sarcoidosis are (1) bilateral hilar and occasionally right paratracheal adenopathy, (2) thoracic lymphadenopathy and pulmonary densities, (3) pulmonary densities without lymphadenopathy, and (4) predominant pulmonary fibrosis.

Occasionally, the clue as to the possible clinical diagnosis of pulmonary sarcoidosis is the presence of bilateral hilar lymphadenopathy. Sarcoidosis is not usually considered in the differential diagnosis of unilateral hilar enlargement or of right paratracheal adenopathy alone, but it should be.

\section{MATERIAL AND FINDINGS}

A review of the chest radiographs in 800 histologically diagnosed cases of sarcoidosis at the Mayo Clinic from 1960 through 1969 disclosed 38 cases in which the initial unbiased radiographic interpretation was unilateral right hilar enlargement (22 patients), left hilar enlargement (12 patients), or right paratracheal mass (4 patients). A right paratracheal mass was noted in six of the patients with unilateral hilar lymphadenopathy; thus, a total of 10 of the 38 patients had right paratracheal adenopathy. Of the 800 patients whose chest radiographs were reviewed, 472 had mediastinal adenopathy. Therefore, approximately $8 \%$ of mediastinal lymphadenopathy presented unilaterally.

The differential diagnosis most commonly included lymphoma or bronchogenic carcinoma and, less frequently, sarcoidosis. The diagnosis was confirmed in all cases; cultures for acid-fast bacilli and fungi were negative in all cases. In retrospect, in some of the radiographs, the opposite hilus appeared suspiciously involved but was not considered so until after the diagnosis had been confirmed histologically. Patients considered initially to have bilateral hilar involvement were not included in this study.

Positive histological confirmation was obtained by scalene node biopsy in 26 cases, by thoracotomy in 8 , by mediastinoscopy in 2 , and by bronchoscopy and muscle biopsy in 1 each. Follow-up radiographs were available from four months to six years in 26 cases. The disease remained stationary in three, progressed to bilateral involvement in three, and regressed to normal in 20. A purified protein derivative test was done in 24 patients, and four had positive reactions. Six patients presented with erythema nodosum.

\section{THREE REPRESENTATIVE CASES}

CASE 1 A 59-year-old man had evidence of a right hilar mass on a routine chest film (Fig. 1a) and was referred to the Mayo Clinic for further evaluation. He was asymptomatic, except for residuals of a recent chest cold, and there were no abnormal findings on physical examination. Results of routine laboratory studies were within normal limits. In retrospect, the left hilus was suspiciously involved but was not considered so until after the diagnosis had been established at thoracotomy. Thoracotomy was preceded by negative bronchoscopy and negative scalene node biopsy. Cultures for acid-fast bacilli and fungi from the removed lymph nodes were also negative. A tomogram of the right hilus showed evidence of the mass (Fig. 1b).

CASE 2 A 56-year-old woman came to the Mayo Clinic for evaluation of an adenomatous goitre. 


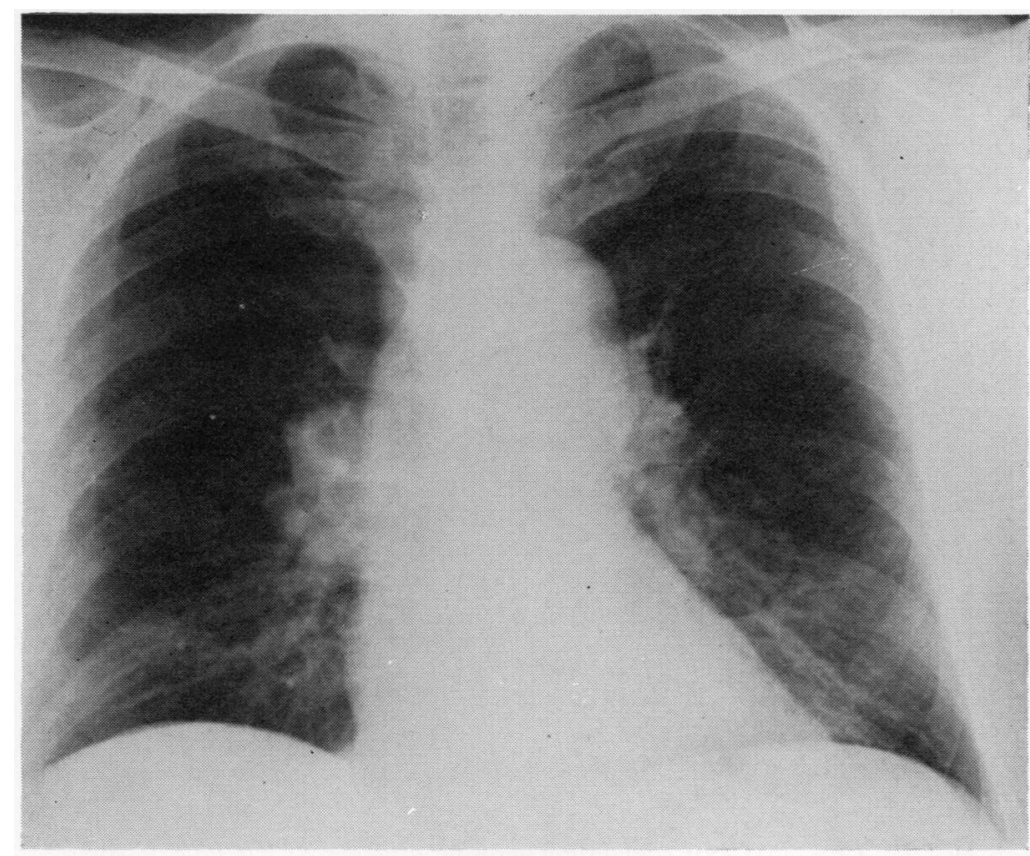

(a)

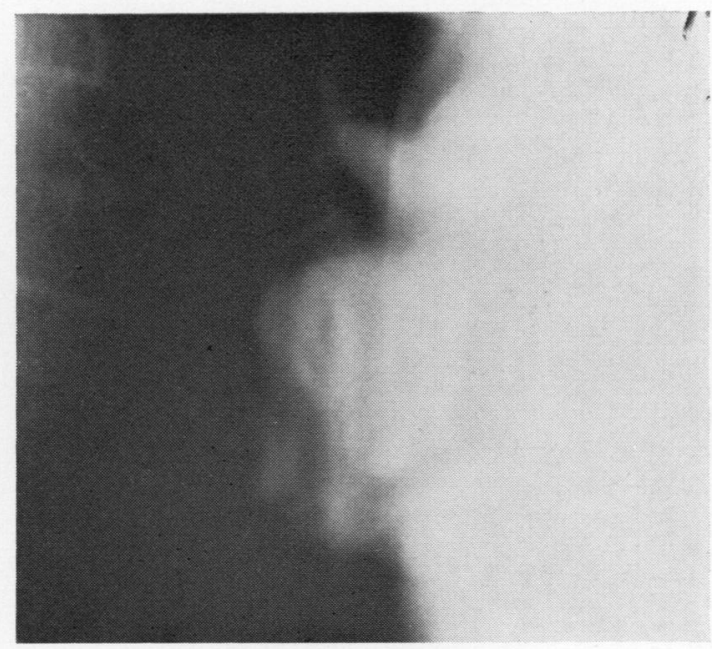

(b)

FIG. 1. Case 1 (a) Chest radiograph showing evidence of right hilar mass. Left hilus, in retrospect, is suspiciously involved. (b) Tomogram of right hilar mass. 


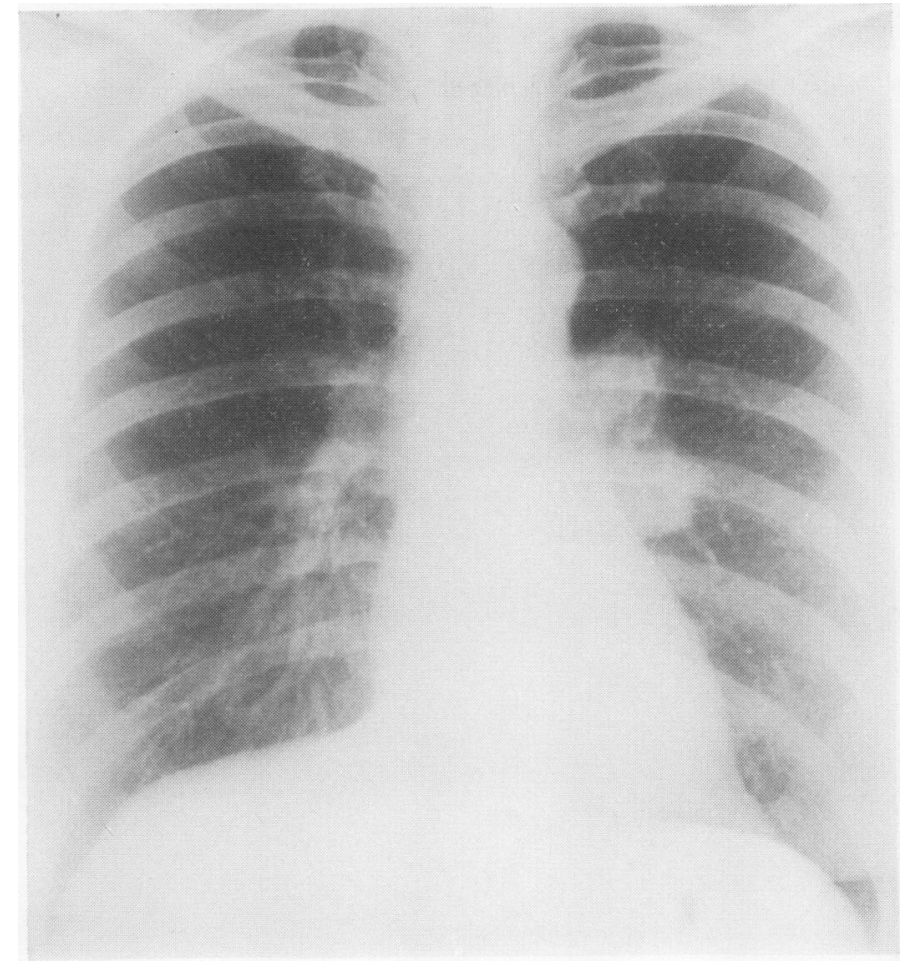

FIG. 3. Case 3. Chest radiograph showing evidence of right paratracheal mass. Lateral chest radiograph disclosed that this mass was in the middle mediastinum. 
Evidence of a left hilar mass was found on a routine chest film (Fig. 2). A colloid goitre was removed at thyroidectomy, at which time the surgeon also removed lymph nodes from the upper mediastinum, which showed non-caseous granuloma. A chest radiograph four months later revealed no change in the left hilar mass.

CASE 3 A 29-year-old woman had evidence of a right paratracheal mass on a chest film taken for evaluation of a mild cough of six months' duration (Fig. 3). Results of physical examination were negative, with the exception of mild hypertension. A low haemoglobin level of $11.5 \mathrm{~g} / 100 \mathrm{ml}$ was considered to be due to an unrelated iron-deficiency anaemia. The serum calcium level was raised on three occasions, being $10 \cdot 4,10 \cdot 1$, and $10 \cdot 5 . \mathrm{mg} / 100 \mathrm{ml}$ (normal 8.9 to $10 \cdot 1 \mathrm{mg}$ ). One year later the serum calcium level was $9.9 \mathrm{mg} / 100 \mathrm{ml}$. Tissue removed at mediastinoscopy showed non-caseous granuloma. All cultures were negative. A purified protein derivative test with 250 tuberculin units and histoplasmin skin tests were negative. A chest radiograph one year later revealed that the mass had almost completely disappeared.

\section{DISCUSSION}

Recently, Kent (1965) stressed the importance of considering sarcoidosis in the differential diagnosis of unilateral hilar lymphadenopathy, along with tuberculosis, lymphoma, bronchogenic carcinoma, and metastat:c carcinoma. His report described a patient in whon unilateral left hilar lymphadenopathy regressed and was followed three years later by development of right hilar lymphadenopathy. Some authors have emphasized that hilar enlargement may be unilateral in sarcoidosis (Gendel, Young, and Greiner, 1952; Kerley, 1956; Longcope and Freiman, 1952 ; Turiaf, 1964). There are many other reports of one to four patients with unilateral hilar involvement (Citron, 1958 ; Hedvall, 1960 ; James, 1961 ; Löfgren, 1953; Miech, Morand, Janser, Reys, and Witz, 1965; Moyer and Ackerman, 1950 ; Nitter, 1953 ; Rudberg-Roos, 1962 ; Scadding, 1950 ; Siltzbach, 1955; Susmano and Carleton, 1970 ; Williams, 1961). Most of these reports are one part of a large series of patients with sarcoidosis. Ellis and Renthal (1962) noted 13 patients with unilateral hilar lymphadenopathy out of 76 patients with hilar lymphadenopathy. In many of the patients bilateral hilar lymphadenopathy subsequently developed.
It is believed that hilar lymphadenopathy is the first stage of development of pulmonary sarcoidosis ; not unlikely, the lympadenopathy begins as or regresses to a unilateral or an asymmetric stage.

\section{REFERENCES}

Citron, K. M. (1958). Intrathoracic sarcoidosis. Postgrad. med. J., 34, 268.

Ellis, K., and Renthal, G. (1962). Pulmonary sarcoidosis: roentgenographic observations on course of disease. Amer. J. Roentgenol., 88, 1070.

Gendel, B. R., Young, J. M., and Greiner, D. J. (1952). Sarcoidosis: a review with twenty-four additional cases. Amer. J. Med., 12, 205.

Hedvall, E. (1960). The prognosis of sarcoidosis. Acta tuberc scand., 39, 249.

James, D. G. (1961). Course and prognosis of sarcoidosis: London. Amer. Rev. resp. Dis., 84, no. 5, Part 2, p. 66.

Kent, D. C. (1965). Recurrent unilateral hilar adenopathy in sarcoidosis. Amer. Rev. resp. Dis., 91, 272.

Kerley, P. (1956). Discussion on sarcoidosis. Proc. roy. Soc. Med., 49, 803.

Löfgren, S. (1953). Primary pulmonary sarcoidosis. I. Early signs and symptoms. Acta med. scand., 145, 424.

Longcope, W. T., and Freiman, D. G. (1952). A study of sarcoidosis based on a combined investigation of 160 cases including 30 autopsies from the Johns Hopkins Hospital and Massachusetts General Hospital. Medicine (Baltimore), 31, 1.

Miech, G., Morand, G., Janser, J., Reys, P., and Witz, J. P. (1965). Les localisations broncho-pulmonaires unilatérales de la maladie de Besnier-Boeck-Schaumann et Pautrier (à propos de 3 observations). J. Radiol. Électrol., 46, 74.

Moyer, J. H., and Ackerman, A. J. (1950). Sarcoidosis: A clinical and roentgenological study of twenty-eight cases. Amer. Rev. Tuberc., 61, 299.

Nitter, L. (1953). Changes in the chest roentgenogram in Boeck's sarcoid of the lungs: A study of the course of the disease in 90 cases. Acta radiol. (Stockh.), Suppl. 105.

Rudberg-Roos, I. (1962). The course and prognosis of sarcoidosis as observed in 296 cases. Acta tuberc. scand., 41, Suppl. 52.

Scadding, J. G. (1950). Sarcoidosis, with special reference to lung changes. Brit. med. J., 1, 745.

Siltzbach, L. E. (1955). Pulmonary sarcoidosis. Amer. J. Surg., 89, 556.

Susmano, A., and Carleton, R. A. (1970). Study of hilar masses by angiocardiography. Chest, 57, 406.

Turiaf, J. (1964). Les inscriptions radiographiques inattendues de la sarcoidose pulmonaire. Rev. Tuberc. (Paris), 28, 971.

Williams, M. J. (1961). Sarcoidosis presenting with unilateral hilar lymph-node enlargement: report of a case. Scot. med. J., 6, 18. 